\title{
Two Dimensional Space Curve Based Curvature Induced Stiffness Formulation for Large Deformable Structural Cables
}

\author{
M. Vignarajah ${ }^{1}$, R. Wijesiriwardana ${ }^{1}$ and P. Kathirgamanathan ${ }^{1}$
}

\begin{abstract}
In this paper, we present a curvature based modification to the existing beam element formulation to model cables which undergoes large displacements. The proposed approximation is numerically tested against the P-delta formulation and true nonlinear formulation of cables. As a result, a limiting value for the curvature based stiffness is reported.
\end{abstract}

Keywords - cables, finite elements, small strains, geometric nonlinartity, large displacements.

\section{Introduction}

In this paper, we present the numerical study of cable finite element using stiffness matrices obtained form beam and string type elements. Both stiffness matrices include large displacements as a second order effect. In practical point of view, cables are modeled with beam elements with reduced bending stiffness and P-delta effect [11]. Cables are highly flexible structural elements. The deformed shape of cables is load dependent.

In the analysis of cable structures, incremental strain formulation with nonlinear capabilities are demanded $[1,3]$. It requires incremental-iterative procedures which requires high computing power and finite element skills. In addition, true nonlinear iso-parametric cable elements with three nodes were developed with non-incremental forms.

Theoretically, cables (such as strings) do not show significant bending moments. The primary load transfer mechanism is through axial deformation that results in heavy axial loads. Therefore, using beam element with Pdelta gives limited corrections to the problem considered $[10,7]$.

This paper examines the limits of using beam element by modifying the bending stiffness into curvature that resulted from the kinematic conditions. This method will release the bending effect and hence include the large displacements as a second order correction.

M. Vignarajah

R. Wijesiriwardana

P.Kathirgamanathan

1 - Faculty of Engineering, University of Jaffna

Sri Lanka.

\section{Kinematics}

\section{A. Displacement field}

Consider a plane cable that undergoes displacements. In this case, assume that material law is linear and small axial strains. It is obvious that cable may undergo large rotations and displacements but the axial strain may be small. Since plane cable is considered, it does not show any torsion in the cable. The deformed location $(\boldsymbol{x})$ of material point is given by the vector sum of original location $(\boldsymbol{X})$ and displacement vector $(\boldsymbol{u})$

$$
\boldsymbol{x}=\boldsymbol{X}+\boldsymbol{u}
$$

The unit tangent vector to the deformed configuration is given by the arc-length $(s)$ derivative of the coordinate vector.

$$
\boldsymbol{t}=\frac{\partial \boldsymbol{x}}{\partial s}
$$

We assume that the normal component of the displacement vector is $u_{t}$ and the strain in the tangential direction is

$$
\epsilon=\frac{\partial u_{t}}{\partial s}
$$

The normal strain, $\epsilon$ is small and higher order terms are neglected.

\section{B. Strain-displacement matrices}

Consider a three node, iso-parametric cable element. The coordinates and displacements are approximated by using the nodal coordinate vector $\left(\boldsymbol{X}^{a}\right)$ and nodal displacement vector $\left(\boldsymbol{d}^{a}\right)$ in the following manner with Einstein summation scheme:

$$
\begin{aligned}
\boldsymbol{x} & =N_{a} \boldsymbol{X}^{a} \\
\boldsymbol{u} & =N_{a} \boldsymbol{d}^{a}
\end{aligned}
$$

Node numbers are given by $a=1,2,3$.

The tangential component of the displacement vector given in the previous section can be written in the following form.

$$
u_{t}=\boldsymbol{u} \cdot \boldsymbol{t}
$$

The normal strain can now be written as follows:

$$
\epsilon=\frac{\partial \boldsymbol{u}}{\partial s} \cdot \boldsymbol{t}+\boldsymbol{u} \cdot \frac{\partial \boldsymbol{t}}{\partial s}
$$

The arc-length derivative of displacement vector can be given in the standard form: 


$$
\frac{\partial \boldsymbol{u}}{\partial s}=\frac{\partial N_{a}}{\partial s} \boldsymbol{d}^{a}=N_{a, s} \boldsymbol{d}^{a}
$$

We use the Frenet-Serret formula for tangent, normal and binormal vectors and the term

$$
\frac{\partial \boldsymbol{t}}{\partial s}=k \boldsymbol{n}
$$

Where $k=$ ccurvature at the specified location.

Now the normal strain in the cable element can be formulated in nodal parameters such that:

$$
\begin{aligned}
\epsilon & =N_{a, s} \boldsymbol{t}^{T} \boldsymbol{d}^{a}+k N_{a} \boldsymbol{n}^{T} \boldsymbol{d}^{a} \\
& =\boldsymbol{B}_{a} \boldsymbol{d}^{a}
\end{aligned}
$$

Therefore, the strain displacement matrix $\boldsymbol{B}_{a}$ can be given by:

$$
B_{a}=N_{a, s} \boldsymbol{t}^{T}+k N_{a} \boldsymbol{n}^{T}
$$

It shows that the value of strain at any point is contributed from tangential displacement and curvature.

The curvature can be obtained from beam bending and/or a space curve. With usual notations, beam bending curvature can be given by

$$
k_{b}=\frac{M}{E I}
$$

Where $M=$ bending moment and

$$
E I=\text { flexural stiffness at the location considered. }
$$

When the cable undergoes large displacements, it can introduce curvature change in the cable profile and that can add additional stiffness to the system. The curvature of space curve can be written in the following form:

$$
k_{s}=\left\|\frac{\partial t}{\partial s}\right\|
$$

Since small strain assumption hold, the effect of such bending and large displacement can be accommodated by denoting

$$
k=k_{b}+k_{s}
$$

The strain-displacement matrix can now be constructed with nodal displacement vectors.

\section{Element Stiffness Matrices}

The element stiffness matrix $\boldsymbol{K}^{e}$ arise from the virtual work form (weak form) or minimum potential energy theory can be readily available in the following manner.

$$
\boldsymbol{K}^{e}=\left[\boldsymbol{k}_{a b}\right]
$$

where $\boldsymbol{k}_{a b}$ is the nodal submatrix.

Nodal submatrices can be estimated using the standard form: (14)

$$
\boldsymbol{k}_{a b}=\iiint_{\Omega} \boldsymbol{B}_{a}^{T} \cdot A E \cdot \boldsymbol{B}_{b} d \Omega
$$

Numerical computing of the strain-displacement matrix requires estimation of unit tangent vector $\boldsymbol{t}$, unit normal vector $\boldsymbol{n}$ and curvature $k$.

\section{A. Beam with P-Delta stiffness}

Plane beam element can take two degrees of freedom per node, namely transverse displacement and rotation. The geometric stiffness (P-delta stiffness) can be obtained from second order effect in the following form, where $P$ is axial load and $L=$ element length [11].

$$
K_{G}=\frac{P}{30 L}\left[\begin{array}{cccc}
36 & 3 L & -36 & 3 L \\
3 L & 4 L^{2} & -3 L & -L^{2} \\
-36 & -3 L & 36 & -3 L \\
3 L & -L^{2} & 3 L & 4 L^{2}
\end{array}\right]
$$

\section{Iv. Numerical Examples}

In this section we consider a typical example of freely hanging cable in vertical plane with unsymmetric loading at the mid span. Then the displacements were evaluated using standard P-delta method, proposed curvature modification method and true nonlinear formulation.

Initial geometry of the cable is a parabolic curve with the coordinates

Table 1: Nodal coordinates of the model

\begin{tabular}{|l|c|c|c|c|c|}
\hline Node & 1 & 2 & 3 & 4 & 5 \\
\hline x-coordinate & 0.0 & 2.0 & 4.0 & 6.0 & 8.0 \\
\hline z-coordinate & 2.0 & 0.5 & 0.0 & 0.5 & 2.0 \\
\hline
\end{tabular}

Undeformed configuration is given in Figure (1).

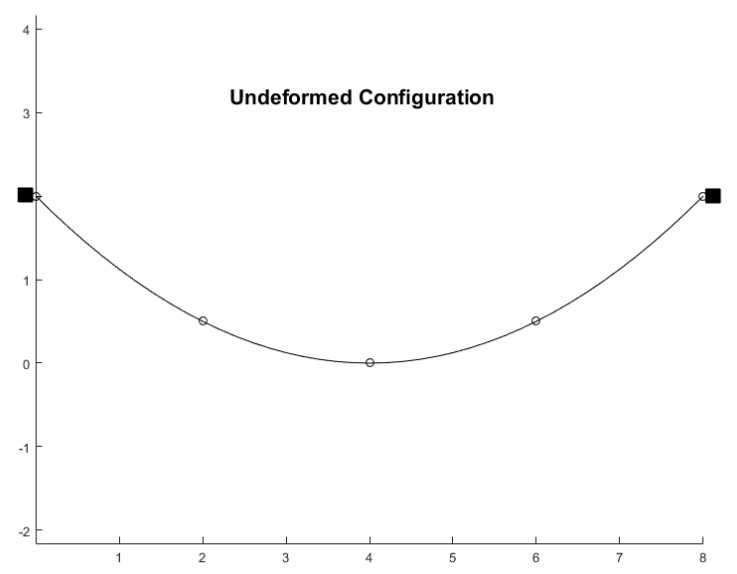

Figure 1: Undeformed shape of cable. (2).

Mechanical properties of the cable are given in Table

Table 2: Mechanical properties of cable material

\begin{tabular}{|l|l|}
\hline Property & Value \\
\hline
\end{tabular}




\begin{tabular}{|l|c|}
\hline Area $\left(\mathrm{m}^{2}\right)$ & $1.0 \mathrm{e}-3$ \\
\hline Young's modulus $(\mathrm{GPa})$ & $200 \mathrm{e} 9$ \\
\hline Moment of inertia $\left(\mathrm{m}^{4}\right)$ & $1.0 \mathrm{e}-7$ \\
\hline
\end{tabular}

A vertically downward load of $10 \mathrm{kN}$ and horizontal load in the negative $\mathrm{X}$-axis is applied at the mid node of the cable.

\section{A. Case 1: Analysis of the system with P-delta method.}

P-delta formulation applies a second order correction to the standard beam finite element [11]. The procedure is an incremental-iterative solution scheme. It is evident that the additional bending moment arises from slight change in geometry when axial loads are high. Specially in cables, the P-delta takes advantage to stiffen the structure if tensile force is applied in the axial direction. Cables are of some examples.

The stiffness resulted from standard beam will be increased by adding appropriate geometric stiffness $\left(K_{G}\right)$ which depends on axial tension. In the solution process, iterative methods are applied until axial loads stabilize.

The problem described in Section IV is modelled by using two node geometrically nonlinear beam elements [12]. In the subsequent analysis, convergence reached and the deformed shape (see Figure 2) and nodal displacement is reported.

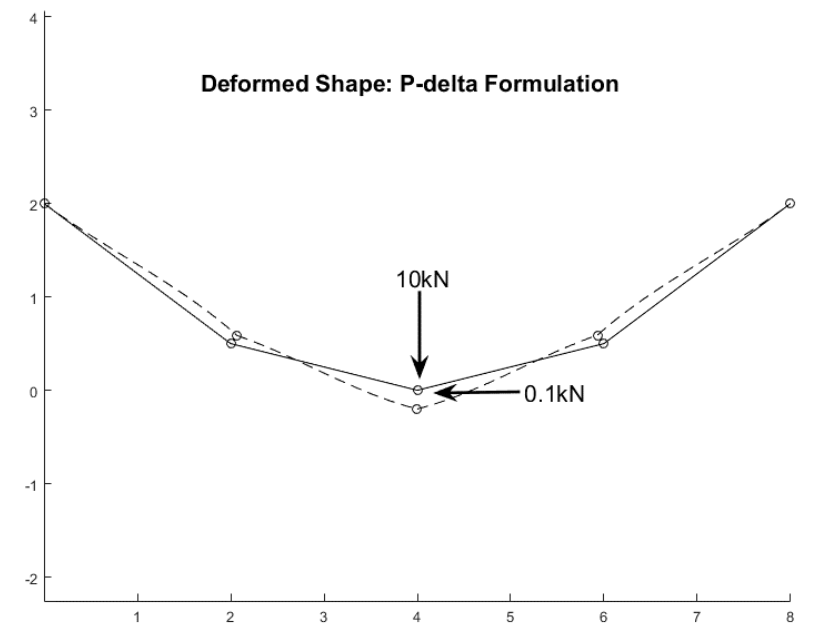

Figure 2: Deformed shape for P-delta analysis

The nodal displacement vector $\boldsymbol{d}^{3}=[0.0000,-0.0090]$ is obtained in the analysis.

\section{B. Case 2: Analysis of the system with curvature stiffness formulation}

The test problem is analyzed with the curvature based stiffness formulation. The strain-displacement array shown in Section II(B) shows that tangential and normal displacement contribute to the development of axial strain. In case of classic beam theory, the curvature is formed by bending the material fibres of the cable. In the current formulation, cable deforms and form the space curve so that the amount of curvature can be estimated for the work done.

The deformed shape and control node displacement are reported below.

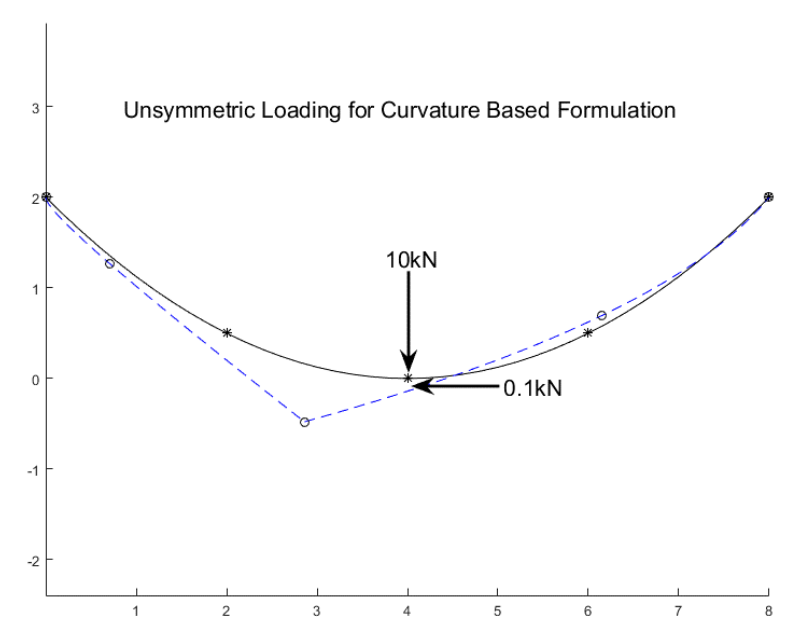

Figure 3: Deformed shape in curvature based form by

Nodal displacement vector of the control node is given

$$
\boldsymbol{d}^{3}=[-0.0760,-0.0319]
$$

\section{Case 3: Analysis of the system with full nonlinear formulation}

A geometrically nonlinear, iso-parametric cable element was developed by considering non-incremental finite element procedures [7]. The nonlinear formulations are expensive and time consuming. Unless otherwise needed to solve for a very complicated problem approximate solution with certain accuracy may be useful. For the comparison, full nonlinear analysis is done. 

reported

Deformed shape and nodal displacement vector are

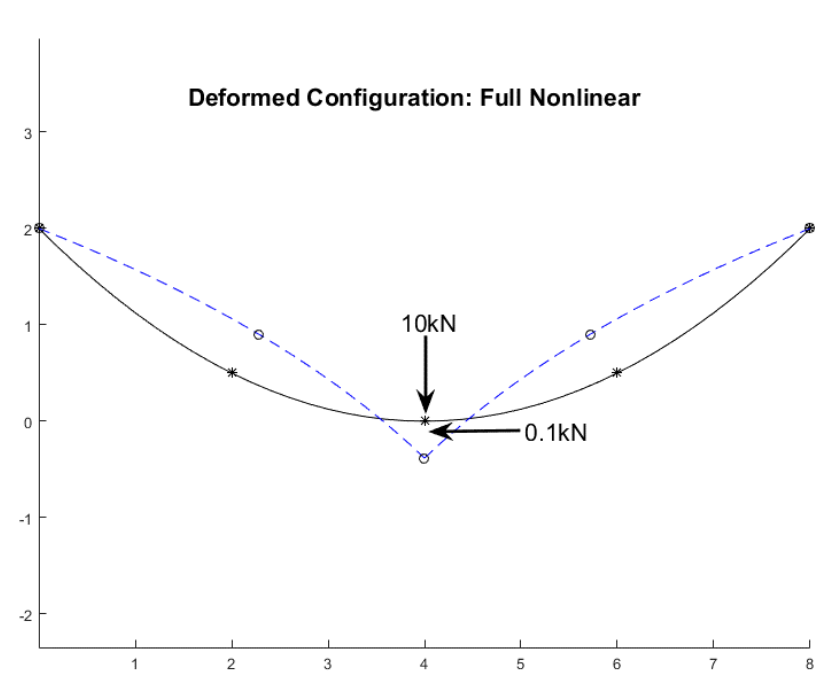

Figure 4: Deformed shape in full nonlinear analysis noted.

Nodal displacement vector $\boldsymbol{d}^{3}=[-0.000,-0.2544]$ is

\section{v. Results and Discussions}

The primary results show that the standard-beam formulation with P-delta method looks like a stiff solution. The reason is that the kinematic conditions assumed in beam formulation does not allow large rotations though strains are small.

In the curvature based formulation, there exist significant rotations. It shows that there is some amount of improvement in the lateral displacements compared with the P-delta formulation.

The full nonlinear analysis gives exact solution which is almost ten times the curvature based formulation. In the nonlinear analysis Green-Lagrange strain is used and it is capable of capturing large rotations.

Further study is necessary to understand the limiting behavior of the strain in the curvature based formulation. The proposed formulation should be checked with different curvatures and loading.

\section{Acknowledgment}

The authors acknowledge the support provided by the academic staff of Faculty of Engineering, University of Jaffna and University of Peradeniya.

\section{References}

[1] T.P. Dreyer and Jan H. Van Vuuren, "A comparison between continuous and discrete modelling of cables bending stiffness", Int Journal of Applied Mathematical Modeling, vol.23, pp.527-541, 1999.
[2] Ever Coarita and Leonardo Flores, "Nonlinear Analysis of Structures Cable - Truss", IACSIT - Int. Journal of Engineeirng and Technology, vol.7, No.3, 2015.

[3] Janislaw Tranowski, "Improved Method of Determining Bending Stiffness of Underground Cables", $9^{\text {th }}$ Int. Conference on Insulated Power Cables, 2015.

[4] Jon Andreas Moseid, "Mathematical Modeling of Cable and Pulley Systems", Thesis, Norwegian University of Science and Technology, 2017.

[5] M. Raoof and T.J. Davies, "Determination of the bending stiffness for a spiral strand", Journal of Strain Analysis for Engineering Designs, Vol. 39, No. 1, 2004.

[6] Anthony Colozza, "Wire and Cable Cold Bending Test", Reported to NASA, Glenn Research Center, 2010.

[7] M. Vignarajah, "Finite Element Nonlinear Analysis of Cables", MPhil Thesis, Faculty of Engineering, University of Peradeniya, Sri Lanka, 2005.

[8] D. Gopinath, G. Shibu and G. Thanigaiyarasu, "Theoretical Estimation of Stiffness of Stranded Cable Subjected to Constrained Bending", Int.J Offshore and Polar Engineering, Vol. 22, No. 4, 2012

[9] Fabien Treyssede, "Vibration analysis of horizontal self-weighted beams and cables with bending stiffness subjected to thermal loads", Int.J Sound and Vibration, 329(9), pp.1536-1552, 2010.

[10] A.M.S. Freire, J.H.O. Negrao, A.V. Lopes, "Geometrical nonlinearities on the static analysis of highly flexible steel cablestayed bridges", Computers and Structures, Vol. 84, pp.2128-2140, 2016.

[11] Edward L. Wilson, "Three dimensional static and dynamic analysis of structures", Computers and Structures, Inc., Berkeley 2002.

[12] CALFEM: Toolbox for computer aided learing finite element method, Lund University, Sweeden.

M. Vignarajah:

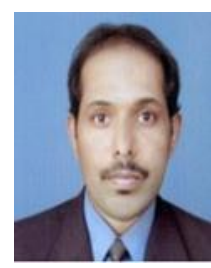

Eng. M. Vignarajah obtained his B.Sc.(Eng) degree with Second Class Honors from the University of Peradeniya in Year 2000. Also he earned M.Phil degree from University of Peradeniya in Year 2005 .

\section{R. Wijesiriwardana:}

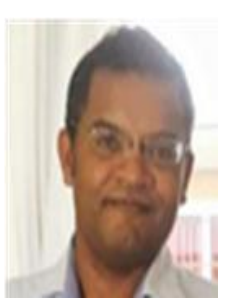

Dr. Ravi is specialized in the multidisciplinary field of wearable physiological information monitoring systems. He received his $\mathrm{Ph} . \mathrm{D}$. from University of Manchester UK from the Faculty of Engineering and Physical Sciences in 2005.

P.Kathirgamanathan:

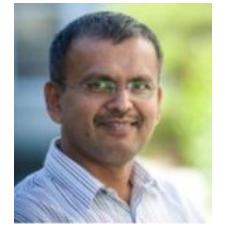

Dr Padmanathan Kathirgamanathan is a senior lecturer with a background in mathematics and engineering. He joined the Faculty in 2015. 